\title{
Safety and feasibility of the PEPPER adaptive bolus advisor and safety system; a randomized control study
}

\section{Authors:}

Parizad Avari $^{1 *}$, Yenny Leal ${ }^{2 *}$, Pau Herrero ${ }^{3}$, Marzena Wos $^{2}$, Narvada Jugnee ${ }^{1}$, María Arnoriaga-Rodríguez ${ }^{2}$, Maria Thomas ${ }^{1}$, Chengyuan Liu $^{3,4}$, Quim Massana ${ }^{5}$, Beatriz Lopez ${ }^{5}$, Lucian Nita $^{6}$, Clare Martin ${ }^{7}$, José Manuel Fernández-Real2,8,9, Nick Oliver ${ }^{1}$, Mercè FernándezBalsells $^{2 \dagger}$, Monika Reddy ${ }^{1 \dagger}$

*These authors contributed equally to this study.

$\dagger$ These authors jointly supervised and guarantee the study.

${ }^{1}$ Imperial College London, Department of Metabolism, Digestion and Reproduction, London, United Kingdom.

${ }^{2}$ Diabetes, Endocrinology and Nutrition Unit, Hospital Universitari Dr. Josep Trueta, Institut d'Investigació Biomèdica de Girona, Girona, Spain.

${ }^{3}$ Imperial College London, Department of Electrical and Electronic Engineering, London, United Kingdom.

${ }^{4}$ University of Nottingham, London, United Kingdom.

${ }^{5}$ University of Girona, eXiT research group, Institut d'Informàtica i Aplicacions, Girona, Spain.

${ }^{6}$ RomSoft SRL, Department of Research \& Development, Iasi, Romania.

${ }^{7}$ Oxford Brookes University, School of Engineering, Computing, and Mathematics, Oxford, United Kingdom.

${ }^{8}$ University of Girona, Faculty of Medicine, Department of Medical Sciences, Girona, Spain.

${ }^{9}$ CIBEROBN Fisiopatología de la Obesidad y Nutrición, Instituto de Salud Carlos III, Spain.

Manuscript word count: 3907 words

\section{Conflicts of Interest: None}

*Preliminary results presented at Advanced Technologies \& Treatments for Diabetes (ATTD) Conference Madrid, Spain-February 19-22, 2020. Abstract ID 680 


\begin{abstract}
$\underline{\text { Abstract }}$
Background: The Patient Empowerment through Predictive Personalised Decision Support (PEPPER) system provides personalised bolus advice for people with Type 1 diabetes. The system incorporates an adaptive insulin recommender system (based on case-based reasoning, an artificial intelligence methodology), coupled with a safety system which includes predictive glucose alerts and alarms, predictive low-glucose suspend, personalised carbohydrate recommendations and dynamic bolus insulin constraint. We evaluated the safety and feasibility of the PEPPER system compared to a standard bolus calculator.
\end{abstract}

Methods: This was an open-labelled multicentre randomized controlled cross-over study. Following 4week run-in, participants were randomized to PEPPER/Control or Control/PEPPER in a 1:1 ratio for 12-weeks. Participants then crossed over after a wash-out period. The primary end-point was percentage time in range (TIR, $3.9 \mathrm{mmol} / \mathrm{L}-10.0 \mathrm{mmol} / \mathrm{L}(70-180 \mathrm{mg} / \mathrm{dL})$ ). Secondary outcomes included glycaemic variability, quality of life, and outcomes on the safety system and insulin recommender.

Results: 54 participants on multiple daily injections (MDI) or insulin pump completed the run-in period, making up the intention-to-treat analysis. Median (interquartile range) age was 41.5 (32.3-49.8) years, diabetes duration $21.0(11.5-26.0)$ years and HbA1c 61.0 (58.0-66.1) $\mathrm{mmol} / \mathrm{mol}$. No significant difference was observed for percentage TIR between the PEPPER and Control groups (62.5 (52.1-67.8) $\%$ vs 58.4 (49.6-64.3) \% respectively, $\mathrm{p}=0.27$ ). For quality of life, participants reported higher perceived hypoglycaemia with the PEPPER system despite no objective difference in time spent in hypoglycaemia.

Conclusions: The PEPPER system was safe but did not change glycaemic outcomes, compared to control. There is wide scope for integrating PEPPER into routine diabetes management for pump and MDI users. Further studies are required to confirm overall effectiveness.

Key Words: Type 1 diabetes, PEPPER, adaptive bolus calculator, artificial intelligence, case-based reasoning, predictive glucose alarms, predictive low-glucose suspend, personalised carbohydrate recommendations.

Clinical trial registration: ClinicalTrials.gov NCT03849755

Figures and table count: 2 figures, 4 tables, 5 supplementary tables 


\section{Introduction}

Insulin bolus calculators can assist people with type 1 diabetes (T1D) to calculate the amount of insulin required for meals to achieve optimal glucose levels (1). Overall, standard bolus calculators have been associated with improved postprandial glucose values, reduced dosing errors, reduction in hypoglycaemia fear and improved confidence in diabetes self-management (2-4). However, HbA1c outcomes have been mixed with some studies showing a reduction (5-7), whilst others show no impact with only improved treatment satisfaction (4). Moreover, some studies have shown improved glycaemic control, but at the expense of increased hypoglycaemia (8).

Whilst some bolus calculators use a rule-based approach to consider parameters such as exercise, illness and menstrual cycle, all commercially available bolus calculators lack the ability to automatically adapt over time to respond to an individual's needs or changes in insulin sensitivity, providing real-time adaptive bolus advice to the user. To overcome this limitation, we propose the use of case-based reasoning $(\mathrm{CBR})$, an established artificial intelligence (AI) methodology $(9,10)$.

CBR addresses the task of solving newly encountered problems by applying the solutions learned from solving similar problems encountered in the past (i.e. cases). Related past cases are stored into a case base, and each case is defined by three components: the problem description, the problem solution and the outcome (10). For example, the problem may be described by parameters affecting glucose levels (such as exercise or alcohol consumption), the solution can be defined by the parameters of a bolus calculator (i.e. insulin sensitivity factor (ISF) and insulin to carbohydrate ratio (ICR)) and the outcome can be the glycaemic metric assessing glucose excursions (11). A CBR system learns by adding each new case in the case-base, after a revision process, making the system adapt its solutions to changes in the individual.

Following the CBR methodology, an adaptive insulin recommender system has been developed and coupled with a safety system in the Patient Empowerment through Predictive Personalised Decision Support (PEPPER) system to provide personalised bolus advice for people with T1D. The safety system is unique to PEPPER and includes a 30-minute glucose forecasting algorithm (12) to provide predictive glucose alerts, as well as predictive low-glucose suspend for pump users (partial and total). In addition, the PEPPER safety system provides personalised carbohydrate recommendations to improve glycaemia, as well as a dynamic bolus insulin constraint. The carbohydrate recommendations refer to when the PEPPER system recommends an oral dose of carbohydrates to correct hypoglycaemia while minimizing rebound hyperglycaemia. This is personalized, based on the participant's weight and "carbohydrate correction factor" is also used which is adaptive. The aim of the dynamic bolus insulin 
constraint module is to eliminate extreme bolus advice. If the recommended insulin dose is outside the limits of ICR $\pm 30 \%$ or ISF $\pm 30 \%$, it is saturated to the corresponding upper or lower bound.

The PEPPER safety system has been previously evaluated and the findings published by Liu et al. $(12,13)$. The PEPPER adaptive insulin recommender system has also been previously published and validated in-silico by Torrent-Fontbona et al (14). Formal usability assessment of the application has also been performed (15). In this study, we clinically evaluate the safety and efficacy of the coupled components.

\section{Study Design and Methodology}

This is an 8-month multi-centred, prospective, randomized controlled study. Participants (on multiple daily injections (MDI) and continuous subcutaneous insulin infusion (CSII; insulin pump) were recruited from two clinical sites; Imperial College London in the UK and the Institut d'Investigació Biomèdica de Girona Hospital Universitari Dr. Josep Trueta (IDIBGI) in Spain. Ethics and device approvals were obtained from the relevant regulatory bodies at each of the sites. All participants provided verbal and written informed consent.

\section{Participants}

Adult participants aged 18 years or older with T1D for at least 1 year using an intensified MDI regimen (defined as a basal bolus regime, not using mixed insulin) or CSII treatment for over 6 months were recruited. Participants had an $\mathrm{HbAlc}$ between $48 \mathrm{mmol} / \mathrm{mol}$ and $86 \mathrm{mmol} / \mathrm{mol}$ with intact awareness of hypoglycaemia. In addition, all participants had completed structured education (either group or 1:1 sessions) and were competent at carbohydrate counting, using insulin to carbohydrate ratios (ICR) and insulin sensitivity factor (ISF) to calculate mealtime insulin boluses.

Participants were excluded if they had an episode of diabetic ketoacidosis (DKA) or severe hypoglycaemia requiring third-party assistance within the preceding 6 months, used regular paracetamol, were pregnant or intending pregnancy, breastfeeding, had active malignancy or endocrinopathy, abnormal renal function or liver cirrhosis, or had a macrovascular complication in the past year.

\section{System Architecture}

The PEPPER architecture has been previously described $(13,16)$. To summarise, the PEPPER system offers a dual architecture to cater for people with T1D on MDI or CSII, the latter via the Cellnovo insulin pump (Figure 1). Participants used the PEPPER user app, which is integrated into a hand-held device. For pump users this was Cellnovo's own handset running an Android OS. For MDI users, this 
was an Android smartphone (Google Nexus 5x). In addition, users wore real-time continuous glucose monitoring (CGM) (Dexcom G5, CA, US) which communicates to the hand-held device via Bluetooth. The CGM data were directly imported through open source software, $x D^{2}$ rip + , into the Android native PEPPER app.

Physical activity prior to a meal was determined through an activity monitor (Mi Band 1s, Xiaomi, China). The activity monitor records the step count performed in the 10 hours prior to the meal. The data are coded into four personalised categories based on each individual's mean step count. The categories included are as follows: "none" <1000 steps, "low" < $(2000+$ individual mean/2), "mild" < $(5500+$ individual mean/2) and "extreme" $>(5500+$ individual mean/2).

Users logged additional lifestyle data such as carbohydrate intake, meal absorption, future physical activity, alcohol consumption, stress, fever, digestive illness and hormone cycle for women (if present), through the user interface of the PEPPER app. The default setting was "none" if not specifically marked.

All collected data, including insulin information, were remotely and automatically uploaded and stored to a remote server via wireless internet connection or mobile data. The web-based user interface (i.e. the PEPPER clinical platform) enables the supervision of the bolus insulin adjustments proposed by the CBR algorithm. Hence, the user is presented with real-time adaptive insulin bolus recommendations on their handset, taking in to account the revised ICR and ISF.

Insulin pen devices with half-unit increments were provided to all participants (Echo pen [Novo Nordisk] for insulin aspart, the Junior Star [Sanofi] for insulin glulisine, or the HumaPen Luxura HD [Lilly] for insulin lispro) as insulin bolus advice was provided to the nearest 0.5 units. When the user accepts insulin recommendations, the data are automatically uploaded into the remote server. If the user rejects the bolus advice, the app requests the user to input the insulin administered. The CBR uses the user insulin dose for revision of cases.

During the run-in and control periods, insulin bolus recommendations were based on a standard bolus calculator using the formula:

$$
B=\frac{C H O}{I C R}+\frac{G_{c}-G_{S p}}{I S F}-I O B
$$

where carbohydrate $(\mathrm{CHO})$ (grams) is the amount of estimated carbohydrate, $\mathrm{G}_{\mathrm{c}}(\mathrm{mmol} / \mathrm{l})$ is the blood glucose measurement pre-meal, $\mathrm{G}_{\mathrm{sp}}(\mathrm{mmol} / \mathrm{l})$ is the blood glucose target, ICR $(\mathrm{g} / \mathrm{U}), \operatorname{ISF}(\mathrm{mmol} / \mathrm{l} / \mathrm{U})$ and IOB (units) is the insulin-on-board. 
The IOB was calculated using linear decay expressed as:

$$
\mathrm{IOB}=\sum_{\mathrm{i}=1}^{\mathrm{N}} \mathrm{Bp}_{\mathrm{i}}\left(1-\frac{\mathrm{T}_{\mathrm{int}_{\mathrm{i}}}}{\mathrm{T}_{\mathrm{act}}}\right)
$$

where $\mathrm{Bp}_{\mathrm{i}}$ is a previously administered insulin bolus and $\mathrm{N}$ is the total number of insulin boluses delivered within the time window $\left[t-T_{\text {act }}, t\right]$, being $t$ the current time and $\mathrm{T}_{\text {act }}$ is the subject-dependent insulin action time; and $\mathrm{T}_{\mathrm{int}_{\mathrm{i}}}$ is the elapsed time since the corresponding insulin bolus $\mathrm{Bp}_{\mathrm{i}}$. Note that only glucose centric insulin calculated from the correction dose are accounted to estimate IOB.

\section{Trial intervention and study procedures}

\section{Visit 1: Consent, training and study enrolment}

At study enrolment, the investigators confirmed the participants' eligibility for the study, obtained written informed consent, recorded demographic information and collected relevant medical and drug history. Study participants underwent electrocardiography, urine analysis, height and weight. Urine and blood samples were collected for laboratory tests ( $\mathrm{HbAlc}$, urinalysis, biochemistry panel with lipids, full blood count and pregnancy testing for women of child-bearing age).

Participants completed validated psychological study questionnaires (Diabetes treatment satisfaction questionnaire (DTSQs), Problem Areas in Diabetes (PAID), and Diabetes Quality of Life (DQOL)).

Participants received the PEPPER study system and completed a 4-week run-in period using real-time CGM (Dexcom G5 transmitter and sensor) to familiarize themselves. For the run-in period, the adaptive insulin recommender and safety system were disabled, and a standard bolus calculator was used. Dexcom G5 sensors were changed every 7 days as per manufacturer's guidance (or sooner in event of sensor failure). Standard CGM alarms were received through xDrip+, which ran in the background of the PEPPER app. Participants were instructed to test their capillary blood glucose every 12 hours for calibration or if symptoms of hypo- or hyperglycaemia, in the event of sensor failure or if the sensor glucose is out of the desired range

Participants on insulin pump therapy were switched to the Cellnovo pump (Cellnovo Ltd, UK) and trained on its use. Differences from their own home pump were discussed including important aspects such as calculation of insulin on board and correction boluses. Additional components of training included: site initiation, cartridge/priming procedures, setting up the pump, navigation through menus, bolus procedures including how to stop a bolus delivery. User manuals for the PEPPER system and the Cellnovo insulin pump were provided. 
Structured education revision was provided, with basal insulin dose, ICR and ISF reviewed and adjusted if required. Participants were requested to obtain bolus advise (via the standard bolus calculator) using the PEPPER handset, as well as use it for any dose correction or additional carbohydrate intake.

\section{Visit 2: Randomization}

At visit 2, enrolled participants were randomized to PEPPER/Control or Control/PEPPER in a 1:1 ratio by an online randomization tool. The groups were stratified by insulin delivery modality (CSII or MDI).

For participants in the PEPPER group, the PEPPER forecasting algorithm and the adaptive insulin recommender were activated remotely by using the PEPPER clinical server. CGM alarms based on the forecasting algorithm (12) and alarms based on actual CGM readings would be received by the user directly through the PEPPER app.

For participants in the Control group, the standard bolus calculator integrated into the PEPPER handset was used, with the adaptive insulin recommender system disabled. The PEPPER safety system was also switched off, but participants in the Control group continued to receive standard CGM alarms (activated through xDrip+ $(17,18))$. Alert and alarm settings were initially standardized, but these could subsequently be altered by participants, except for the hypoglycaemia alarm which was hard-coded at $3.9 \mathrm{mmol} / \mathrm{L}(70 \mathrm{mg} / \mathrm{dL})$ due to safety reasons. Alert thresholds for hypoglycaemia and hyperglycaemia were $4.4 \mathrm{mmol} / \mathrm{L}(80 \mathrm{mg} / \mathrm{dL})$ and $14.0 \mathrm{mmol} / \mathrm{L}(250 \mathrm{mg} / \mathrm{dL})$ respectively.

Target blood glucose on the PEPPER bolus calculator was standardized to $5.5 \mathrm{mmol} / \mathrm{L}(99 \mathrm{mg} / \mathrm{dL})$, however this target could subsequently be individualised if needed.

Participants were able to contact the study team for clinical and technical support. Where necessary, glucose data could be reviewed remotely by the PEPPER clinical team.

\section{Visit 3: Review}

Any technical issues were reviewed at the visit, and adjustments to settings made if required.

\section{Visit 4: end of intervention phase 1}

Following completion of 3 months in the intervention phase, each group reverted to their standard therapy for 3-4 weeks (wash-out period). They then crossed over to the other group. Non-fasting venous blood samples were taken for HbAlc, weight and basal insulin requirements were recorded, as well as study questionnaires completed. If participants were in the PEPPER group first, they were not aware of 
their ICR and ISF values at the end of the intervention phase, and therefore reverted back to their initial parameters from the run-in period.

\section{Visit 5-7: start of intervention phase 2}

Visits 5-7 follow the same structure as Visits 2-4. Participants switch on the PEPPER device and are crossed over from PEPPER/Control to Control/PEPPER. For participants crossing-over to the PEPPER intervention arm second, the adaptive insulin recommender system with CBR was initialised at the start of the second PEPPER phase. Thus, the case-base was built up over the intervention period (12 weeks) only for each participant.

At the end of the study (i.e. visit 7 - end of 8 months), repeat blood tests to assess $\mathrm{HbA} 1 \mathrm{c}$ were performed and questionnaires similar to that at baseline and visit 4 were completed.

\section{PEPPER case base revision}

The PEPPER case base was revised by clinicians twice weekly throughout the intervention period (PEPPER with CBR). This was a semi-automated process completed remotely via the secure webserver with all data automatically uploaded. The reviewing clinician rejected case revisions by the CBR algorithm if a meal or insulin bolus was taken within the 5-hour post prandial period; if CGM sensor data quality affected the post-prandial glucose curve; if IOB was $>4$ units, or if the carbohydrate content of a meal was $<20 \mathrm{~g}$ (i.e. snack).

Once cases were accepted, these would be stored in the CBR case base for future use. When users request an insulin dose recommendation, the CBR algorithm looks for a similar case in the case base and computes the recommended dose based on the retrieved ICR and ISF, which is then presented on the user's handset. Then, the CBR creates a new case and revises the glycaemic outcome corresponding to the recommended dose by analysing the post-prandial CGM curve. If the outcome is sub-optimal (i.e. above or below glucose target range), then the new ICR and ISF are revised accordingly. A comprehensive description of the PEPPER CBR process and algorithms, including methods for case retrieval, reuse and revision, has been previously published (14).

The parameters used by the CBR algorithm to determine similar cases were time of day, meal absorption rate, physical activity, alcohol quantity, tiredness, stress, fever, digestive illness and menstrual cycle for women. Past physical activity was recorded through the activity monitor and future activity was based on the user log.

\section{Primary and Secondary Outcomes}


The primary outcome was the difference in change in percentage (\%) time in range (TIR) $(3.9-10.0$ $\mathrm{mmol} / \mathrm{L}(70-180 \mathrm{mg} / \mathrm{dL}))$ between the intervention arm that receives the PEPPER safety system with adaptive bolus advice, and the Control arm. Endpoint assessment was not blind to the study intervention arm allocated.

Secondary endpoints were \% time spent in euglycaemia $(3.9-7.8 \mathrm{mmol} / \mathrm{L} ; 70-140 \mathrm{mg} / \mathrm{dL})$, hypoglycaemia ( $<3.9 \mathrm{mmol} / \mathrm{L} ;<70 \mathrm{mg} / \mathrm{dL}$ ) and hyperglycaemia ( $>10.0 \mathrm{mmol} / \mathrm{L} ;>180 \mathrm{mg} / \mathrm{dL})$, number of episodes of serious hypoglycaemia (defined as a sensor glucose $<3.0 \mathrm{mmol} / \mathrm{L} ;<55 \mathrm{mg} / \mathrm{dL}$ ) for $>20$ min, episodes of hypoglycaemia within 5-hour postprandially, severe hypoglycaemia (defined as a hypoglycaemia event requiring third-party assistance), postprandial mean area under the curve (AUC) at 5 hours (expressed as $\mathrm{mmol} / \mathrm{L} \mathrm{min}$ ) and glycaemia risk and variability measures.

Evaluated glycaemic variability measures are standard deviation (SD), coefficient of variation (CV), mean amplitude of glycaemic excursions (MAGE), continuous overall net glycaemic action (CONGA), mean of daily differences (MODD), lability index (LI), glycaemic variability percentage (GVP), mean absolute glucose change per unit time (MAG), glycaemic risk assessment diabetes equation (GRADE), M-value, average daily risk range (ADRR), J-index, personal glycaemic status (PGS), index of glycaemic control (IGC), risk index (RI), low blood glucose index (LBGI), and high blood glucose index (HBGI). GRADE score is also reported as \%GRADE hypoglycaemia, \%GRADE euglycaemia, and \%GRADE hyperglycaemia representing percentages of GRADE scores attributable to glucose values $<3.9 \mathrm{mmol} / \mathrm{L}(<70 \mathrm{mg} / \mathrm{dL})$, and between $3.9-7.8 \mathrm{mmol} / \mathrm{L}(70-140 \mathrm{mg} / \mathrm{dL})$ and $>7.8 \mathrm{mmol} / \mathrm{L}(>140 \mathrm{mg} / \mathrm{dL}$ ) respectively. Measures of glycaemic variability were computed using EasyGV (v10.0) software (19).

Other secondary endpoints were $\mathrm{HbAlc}$, change in weight $(\mathrm{kg})$, basal insulin dose requirements and diabetes-specific measures of quality of life (PAID, DQOL, DTSQs). Safety system outcomes were also assessed for incidence of low and high glucose alarms, alerts, carbohydrate recommendations, lowglucose suspend and bolus insulin saturation. Outcome measures of the insulin recommender included the number of bolus recommendations, and proportions of those that were accepted or rejected by user. The number of cases revised by clinicians were also evaluated.

\section{Statistical considerations}

Glycaemic outcome measures were analysed at baseline (for 28 days during the run-in period) and using data from the last 28 days from each intervention period. Changes from baseline, and between group differences were assessed. The primary analysis was conducted using the intention-to-treat (ITT) principle. To be included in the ITT analysis, participants had to have completed the full 28-day run-in 
period. A sub-analysis was also done to evaluate differences in outcomes between the pump and MDI groups.

Analysis was also then completed with the per protocol population (PPP), which consisted of those participants in the ITT population who completed the study with no significant deviations from the planned protocol procedures. The study was terminated early for select pump participants due to the withdrawal of pump manufacturer, Cellnovo, withdrawing from market and insufficient supplies. For participants to be included in the PPP analysis, they had to have fully completed the study (both control and intervention phases) and had to have used the PEPPER insulin recommender system. Results from all the sub-analysis can be found in the supplementary material.

Safety system measures were reported for the PEPPER and Control groups. Whilst the safety system was not enabled in the Control group, the algorithm was running in the background to allow comparisons to be made. Data from the run-in period for the safety system was not used in the analysis because the algorithm was optimized using the run-in data itself.

For outcomes related to the adaptive insulin recommender, these have been reported for the full PEPPER intervention period (i.e. 12 weeks) to reflect the number of cases that contributed to the final glycaemic outcome data.

The data were tested for normal distribution using quantile plots and the Shapiro-Wilk test of normality. Differences between the PEPPER and Control groups were tested for significance using the Wilcoxon Rank sum test. For the PPP analysis, the Wilcoxon matched-pairs signed-rank was used for analysis for differences between the intervention groups. Where appropriate, the nonparametric Spearman rank tests was performed for correlation.

Data have been presented as medians (interquartile ranges (IQR), unless otherwise stated. Statistical tests were two tailed and results were considered statistically significant if $\mathrm{P}<0.05$. Statistical analysis was performed using Stata version 15 (StataCorp, College Station, Texas).

With 50 participants (25 participants on MDI and 25 participants on CSII) a 0.57 SD difference can be demonstrated as significant with $\alpha$ of 0.05 and $80 \%$ power (two-tailed). Based on a previous pilot study (unpublished data) which showed a population mean (SD) \% time in range (3.9-10 mmol/1) of 61.6 $(18.8) \%$, we aimed to detect a $10.7 \%$ (i.e. $=0.57 \times 18.8$ ) difference in $\%$ TIR to demonstrate significance between the intervention and control in this study. Assuming that about 10\% of participants would drop out, we calculated that a sample size of 55 participants would be needed. 
This sample size is deemed comparable to other technology transfer studies, and is a realistic number for recruitment, providing robust safety data for a new technology. Glycaemic variability and outcome measures on the safety system and insulin recommender were secondary, and therefore the study was not powered to detect statistical differences in secondary outcomes between groups.

\section{$\underline{\text { Results }}$}

\section{Baseline Demographics}

Fifty-eight participants were enrolled, of which 54 participants completed the run-in period, making up the ITT study population ( 3 declined to participate, 1 did not meet inclusion criteria due to high $\mathrm{HbA1c}$; Figure 2). Twenty-six participants $(48.1 \%)$ were male and 28 (51.9 \%) were female. The median (interquartile range) age was 41.5 (32.3-49.8) years with diabetes duration of 21.0 (11.5-26.0) years and $\mathrm{HbAlc}$ of $61.0(58.0-66.1) \mathrm{mmol} / \mathrm{mol}$. Baseline characteristics of the participants are summarized in Table 1. There was no statistical difference between groups at baseline.

Following enrolment, a further 4 participants withdrew. Fifty participants were randomised to PEPPER $(n=24)$ or Control $(n=26)$. Four participants then withdraw (PEPPER $(n=2)$ and Control $(n=2))$ and 1 was withdrawn due to serious adverse event. In total, the first crossover phase was completed by 45 participants (22 PEPPER, 23 Control). Ten participants were withdrawn before the second crossover phase due to loss of Cellnovo device support (PEPPER $(n=5)$ and Control $(n=5))$. One further participant withdrew due to a serious adverse event and 17 participants crossed-over to PEPPER and 17 to Control. All $(n=34)$ completed the second intervention phase. In total, for the ITT, 39 participants allocated to PEPPER and 40 allocated to Control completed the intervention. For the PPP analysis, 1 participant was withdrawn for not using the PEPPER adaptive bolus advisor $(n=1)$. Thus, 33 participants fully completed both intervention phases and used the PEPPER adaptive insulin recommender system, making up the PPP analysis. The flow diagram (Figure 2) summarises recruitment and withdrawals.

\section{Glycaemic outcomes}

For the primary outcome, no difference in \% TIR 3.9-10.0 mmol/L $(70-180 \mathrm{mg} / \mathrm{dL})$ with the PEPPER system compared to Control (62.5 (52.1-67.8) \% vs 58.4 (49.6-64.3) \%) was observed between the two groups ( $\mathrm{p}=0.27$; Table 2 ). For clinically significant hypoglycaemia, as well as $\%$ time in hyperglycaemia for thresholds $>10.0 \mathrm{mmol} / \mathrm{L}(>180 \mathrm{mg} / \mathrm{dL})$ and $>15 \mathrm{mmol} / \mathrm{l}(>270 \mathrm{mg} / \mathrm{dL})$, no significant differences were observed between the PEPPER and Control groups. 
In the PPP analysis, similar observations were noted to the ITT cohort, with no significant differences observed between the PEPPER system compared to Control. PEPPER demonstrated +4.2 (-4.9 to 10.4) $\%$ TIR compared to Control +1.2 (-7.2 to 7.3), $\mathrm{p}=0.10$ (Supplementary Material Table 1).

In the sub-analysis based on modality, the pump participants observed greater increase in \% TIR compared to the MDI cohort, although there were no significant between group differences (Supplementary Material Table 2). No significant differences were observed for glycaemic outcomes stratified by day $(07.00 \mathrm{hrs}-23.00 \mathrm{hrs})$ and night $(23.00 \mathrm{hrs}-07.00 \mathrm{hrs})$, or by country of recruitment (UK vs Spain; Supplementary Material Tables 3 and 4). In addition, the PEPPER system with predictive low glucose suspend was not associated with improved nocturnal hypoglycaemia for pump users. Nocturnal \%TIR 3.9-10.0 mmol/L (70-180 mg/dL) with the PEPPER intervention was $60.8 \%$ (49.8 $65.4) \%$ vs Control 55.6\% (45.3 - 67.6) \% ( $\mathrm{p}=0.66$ ). For clinically significant hypoglycaemia, nocturnal $\%$ TBR $<3.0 \mathrm{mmol} / \mathrm{L}(<54 \mathrm{mg} / \mathrm{dL})$ with the PEPPER intervention was $0.1 \%(0.0-0.4) \%$ vs Control $0.3 \%(0.0-1.6) \%(\mathrm{p}=0.18)$.

\section{Glycaemic variability and other secondary outcomes}

For GV measures, there were no significant differences between the PEPPER and Control groups (Table 2). The number of episodes of hypoglycaemia declined from baseline for both groups, but between the PEPPER and Control groups, no significant differences were observed.

For AUC, post-prandial glucose or HbA1c, there were no differences between the two groups. Body weight and basal insulin did not significantly change between the treatment groups or from baseline.

\section{Quality of Life}

At baseline, psychological questionnaires demonstrated participants had a good quality of life without significant diabetes distress. The median PAID score at baseline was 18.8 (11.3-31.3). No significant differences were observed for diabetes distress, overall diabetes treatment satisfaction, the global quality of life scores nor its subsections. Within the DTSQs questionnaire, there was an increase in perceived frequency of hypoglycaemia with PEPPER in comparison with the Control group $(\mathrm{p}=0.03$, Table 3). This persisted in the PPP analysis ( $<<0.01$; Supplementary Material Table 5).

\section{Safety System Outcomes}

No safety data was obtained from the handset of one participant. For evaluation of the safety system, although the safety system was not enabled in the Control group, the algorithm was running in the background without effect. Safety system outcomes were reported for the last 28 days of each intervention phase. 
No significant differences were observed between groups for number of alerts, alarms, and carbohydrate recommendations (Table 4). Similarly, for pump users the numbers of automated pump suspensions (total and partial) were not significantly different between the two groups. The total bolus insulin per participant per day also remained similar in the two groups: 32.8 (23.9-53.5) units/day in the Control group vs 32.6 (22.1-51.0) units/day in the PEPPER group ( $\mathrm{p}=0.96)$.

The root mean square error (RMSE) of the 30-minute predictive glucose forecasting PEPPER algorithm (12) was $1.6(1.4-1.9) \mathrm{mmol} / \mathrm{L}(29.0(25.3-34.2) \mathrm{mg} / \mathrm{dL})$. The overall mean absolute relative difference (MARD) between the forecasting algorithm and the actual sensor glucose was 14.4 (12.6-15.9) \%.

A total of 303 revisions were constrained and saturated by the safety system, i.e. $5(2-14) \%$ of all proposed bolus recommendations. Of these, $85 \%$ were for hyperglycaemia.

\section{Adaptive Insulin Recommender Outcomes}

During the full PEPPER intervention period (i.e. 12 weeks), a total of 14,723 cases of meal bolus recommendations were imported to the revision platform, with an average of 4.0 (3.3-5.5) cases per participant/day. There was no decay in the number of bolus recommendations requested per day over the study period. A full analysis of the adaptive insulin recommender outcomes and individual case parameter usage can be found in Supplementary Material Table 6.

Out of all imported bolus advice, 46 (26-58) \% of cases (i.e. meal scenarios) were approved by the physician and uploaded to the online platform to be integrated into the learnt case-base. The remaining cases were manually or automatically rejected by the system based on the criteria stated above in the methods.

The majority of bolus recommendations were accepted by each participant, 87 (65-96) \%. For bolus recommendations that were rejected by the user, the proportion of doses reduced (from the total recommendations provided) were $59(28-84) \%$. The median absolute difference in dose was $1.5(1.0-$ 2.0) units.

No correlation was observed between the number of cases per participant and the \%TIR spent by participants $(\mathrm{r}=0.371, \mathrm{p}=0.15)$.

\section{Adverse events}

There were two serious adverse events in the Control group; one participant had an episode of severe nocturnal hypoglycaemia. Another participant developed mild diabetic ketoacidosis requiring hospital admission, which was precipitated by denatured insulin with urinary tract infection. All resolved 
without adverse sequelae. Two other adverse events were reported. These included appendicitis requiring appendicectomy and a fall resulting in fractured fifth metatarsal. None were deemed related to trial intervention with PEPPER safety system or AI algorithm.

\section{$\underline{\text { Discussion }}$}

This study demonstrates proof of concept, safety and feasibility of the PEPPER system with an AIderived adaptive bolus calculator for people with T1D. While all times in ranges moved towards improvement in the intervention group, none of the differences observed between the PEPPER and Control arms reached statistical significance.

To our knowledge, this is the largest randomized controlled trial reporting clinical outcomes of a personalised, adaptive bolus calculator for use with both MDI and CSII therapy. Unfortunately, the study had to be terminated early due to Cellnovo withdrawing from the market. Ten pump participants in the UK had to be withdrawn prematurely as no further pump supplies were available, limiting the power and outcomes of the study. Whilst no final conclusions about the impact of the adaptive intervention can be drawn from the study outcomes, the results are encouraging in moving towards improvement in all outcomes measures for times in range and glycaemic variability.

The change in outcomes compared to baseline may in part be attributable to the effect of CGM alone but this effect is mitigated by the crossover study design. A high early drop-out rate was observed, and one of the key reasons stated was the complexity of the system requiring multiple components. Individuals were required to carry a handset (an additional device to their own mobile handset), CGM, an activity monitor and glucometer, as well as for pump participants requiring additional pump supplies. This reflects the nature of the intervention in a clinical investigation environment while a final implementation can be achieved on a single handset which may be the user's smartphone. Other reasons for high early drop-out rates, which were observed mostly in participants using CSII, were due to challenges with the Cellnovo pump, in particular frequent cartridge ejection or wireless signal loss.

In terms of quality of life, participants reported higher perceived hypoglycaemia with the PEPPER system despite no objective difference in time spent in hypoglycaemic ranges. This did not affect overall diabetes treatment satisfaction; however, it may be a result of the additional forecasted hypoglycaemia alerts received whilst using the PEPPER system. Overall the recruited participants had good baseline glycaemia with good quality of life, without significant diabetes distress which may limit differences emerging between the two groups. 
Limitations associated with the PEPPER CBR-based insulin recommender include the potential need for additional time required for the adaptive insulin recommender system to be effective. The algorithm is likely to be most beneficial to individuals maintaining regular work patterns, rather than shift workers. In the study two shift workers were included, and they may not have derived the benefits of the learning algorithm. The algorithm only adapts for bolus insulin, and assumes that the basal insulin has been optimised. Alternative methods in basal insulin adaptation could potentially be implemented (20). Furthermore, the system is dependent on meal scenarios where the user has not ingested a significant snack or taken an insulin bolus correction within 5 hours of a meal for revision. This may particularly be an issue for individuals tending to snack, or eat their meal in phases. For pump users, the bolus advisor is unable to provide extended phase or dual wave bolusing, which is otherwise incorporated into most insulin pumps and is a clear development requirement for adaptive bolus calculators.

Currently the PEPPER CBR adaptive insulin recommender system is dependent on manual revisions using the online platform. With clear rejection criteria defined, this process can be automated; something that would be required for adoption at scale for people living with T1D.

Other adaptive bolus insulin recommender systems include the Advanced Bolus Calculator for Diabetes (ABC4D) project also uses CBR for its adaptive algorithm. Reddy et al published a 6-week prospective non-randomized single arm pilot study with 10 adult participants using MDI. More than a two-fold reduction in the number of postprandial hypoglycaemic episodes was observed, however the study was not sufficiently powered to show significance (21). Currently, a randomized controlled trial over 8 months with 40 participants is underway (NCT03963219). Tyler et al (2020) evaluated the K-nearest neighbours decision support system (KNN-DSS) recommender engine with physician advice within a feasibility study of 16 people with T1D over 4 weeks (22). An improvement in overnight hypoglycaemia was observed, however this was also associated with a significant reduction in time in target range overnight $(\mathrm{p}=0.034)$. It is interesting to note that for prandial mealtime dosing, a reduction in hypoglycaemia during the daytime was not observed (22). This system is currently being evaluated in a larger clinical trial integrated within a mobile app called DailyDose (23).

Adaptive basal and bolus insulin recommender systems are also being developed. Breton et al assessed University of Virginia Decision Support System (UVA DSS) in a prospective study of 24 subjects with T1D using either CSII or MDI therapy with carbohydrate counting (24). Participants completed two randomized crossover 48-h visits with either usual care or UVA DSS, which provided automated insulin titration, bolus calculation, and carbohydrate treatment advice. The study found that use of the DSS significantly reduced hypoglycaemia, as well as glycaemic variability (24). Another automated decision support system is the DreaMed Advisor Pro (DreaMed Diabetes Ltd, Petah Tikva, Israel), which is the first approved system by the FDA for use in people with T1D on insulin pump therapy, and uses MD- 
Logic. The Advisor Pro has been tested clinically in a single centre feasibility study with 13 participants. No difference was observed for time spent in range or hypoglycaemia (25). The algorithm effectiveness is being further tested in a randomized controlled trial to fully evaluate the system (NCT03003806).

Despite the limitations of the PEPPER study, the data are promising and suggest that, in a powered study over a sufficient period of time, an adaptive bolus advisor may be able to improve glycaemic outcomes. There is wide scope for integrating the PEPPER system into routine diabetes management for both insulin pump users and those on MDI, and the recent availability of connected insulin delivery pens and wearable sensor technology may further enable integration of devices. Worldwide, most people with T1D use MDI regimes and for the foreseeable future, closed-loop control may not be an option for everyone. Systems using AI integrated into an app, such as PEPPER, may enable personalised decision support and may be additionally used alongside automated insulin delivery in future systems to improve times in ranges around the challenges of food and activity.

\section{Conclusion}

In conclusion, the PEPPER system was safe but did not change glycaemic outcomes, compared to control. A larger powered randomized controlled trial is required to confirm overall effectiveness.

\section{Funding:}

This project has received funding from the EU Horizon 2020 programme, grant agreement No. 689810 .

\section{Role of the funding source:}

The funder of the study had no role in study design, data collection, data analysis, data interpretation, or writing of the report. The corresponding author had full access to all the data in the study and had final responsibility for the decision to submit for publication.

\section{Acknowledgments:}

The authors wish to thank all the study participants for their valuable time and commitment. Infrastructure support in the UK was provided by the NIHR Imperial Biomedical Research Centre and the NIHR Imperial Clinical Research Facility. The views expressed are those of the author(s) and not necessarily those of the NHS, the NIHR or the Department of Health and Social Care. Infrastructure support in Spain was provided by the Diabetes, Endocrinology and Nutrition Unit (UDEN), Hospital Universitari Dr Josep Trueta, Institut Català de la Salut. The authors would like to thank Wifredo Ricart, Lidia Sojo, Elisabet Costa, Eduardo Esteve, who helped with the recruitment of participants, as well as 
the nursing and clinical staff of the UDEN, and particularly to Oscar Rovira (CIBEROBN) and Emili Loshuertos, for their work.

The work outlined in this manuscript was developed with the support of the awarded distinction by the Generalitat de Catalunya 2017 SGR 1551, and the European Union's Horizon 2020 research and innovation programme under grant agreement No 689810 (PEPPER).

\section{Disclosures:}

M.R. has received honoraria for advisory board participation from Dexcom and Roche Diabetes. N.O. has received honoraria for speaking and advisory board participation from Abbott Diabetes, Dexcom, Medtronic Diabetes, and Roche Diabetes.

\section{$\underline{\text { References }}$}

1. Schwartz FL, Marling CR. Use of Automated Bolus Calculators for Diabetes Management. Eur Endocrinol. 2013;9(2):92-5.

2. Shashaj B, Busetto E, Sulli N. Benefits of a bolus calculator in pre-and postprandial glycaemic control and meal flexibility of paediatric patients using continuous subcutaneous insulin infusion (CSII). Diabet Med. 2008/10/22. 2008;25(9):1036-42.

3. Ramotowska A, Golicki D, Dzygało K, Szypowska A, Dzygalo K, Szypowska A. The effect of using the insulin pump bolus calculator compared to standard insulin dosage calculations in patients with type 1 diabetes mellitus - systematic review. Exp Clin Endocrinol Diabetes. 2013/01/19. 2013;121(5):248-54.

4. Schmidt S, Meldgaard M, Serifovski N, Storm C, Christensen TM, Gade-Rasmussen B, et al. Use of an Automated Bolus Calculator in MDI-Treated Type 1 Diabetes: The BolusCal Study, a randomized controlled pilot study. Diabetes Care. 2012;35(5):984-90.

5. Ziegler R, Cavan DA, Cranston I, Barnard K, Ryder J, Vogel C, et al. Use of an Insulin Bolus Advisor Improves Glycemic Control in Multiple Daily Insulin Injection (MDI) Therapy Patients With Suboptimal Glycemic Control: First results from the ABACUS trial. Diabetes Care. 2013;36(11):3613-9.

6. Charpentier G, Benhamou PY, Dardari D, Clergeot A, Franc S, Schaepelynck-Belicar P, et al. The Diabeo Software Enabling Individualized Insulin Dose Adjustments Combined With Telemedicine Support Improves HbAlc in Poorly Controlled Type 1 Diabetic Patients: A 6month, randomized, open-label, parallel-group, multicenter trial (TeleDiab 1 Study). Diabetes Care. 2011;34(3):533-9. 
7. Hommel E, Schmidt S, Vistisen D, Neergaard K, Gribhild M, Almdal T, et al. Effects of advanced carbohydrate counting guided by an automated bolus calculator in Type 1 diabetes mellitus (StenoABC): a 12-month, randomized clinical trial. Diabet Med. 2016/10/21. 2017;34(5):708-15.

8. Garg SK, Bookout TR, McFann KK, Kelly WC, Beatson C, Ellis SL, et al. Improved glycemic control in intensively treated adult subjects with type 1 diabetes using insulin guidance software. Diabetes Technol Ther. 2008/08/22. 2008;10(5):369-75.

9. Pesl P, Herrero P, Reddy M, Xenou M, Oliver N, Johnston D, et al. An Advanced Bolus Calculator for Type 1 Diabetes: System Architecture and Usability Results. IEEE J Biomed Heal informatics. 2016;20(1 PG-11-7):11-7.

10. Aamodt A, Plaza E. Case-based reasoning: Foundational issues, methodological variations, and system approaches. AI Commun. 1994;7:39-59.

11. Herrero P, Pesl P, Bondia J, Reddy M, Oliver N, Georgiou P, et al. Method for automatic adjustment of an insulin bolus calculator: in silico robustness evaluation under intra-day variability. Comput Methods Programs Biomed. 2015;119(1 PG-1-8):1-8.

12. Liu C, Vehí J, Avari P, Reddy M, Oliver N, Georgiou P, et al. Long-term glucose forecasting using a physiological model and deconvolution of the continuous glucose monitoring signal. Sensors (Switzerland). 2019;19(19).

13. Liu C, Avari P, Leal Y, Wos M, Sivasithamparam K, Georgiou P, et al. A Modular Safety System for an Insulin Dose Recommender: A Feasibility Study. J Diabetes Sci Technol. 2020;14(1):8796.

14. Torrent-Fontbona F, Lopez B. Personalized Adaptive CBR Bolus Recommender System for Type 1 Diabetes. IEEE J Biomed Heal Informatics. 2019;23(1):387-94.

15. Martin C, Aldea A, Alshaigy B, Avari P, Duce D, Fernández-Balsells M, et al. Application of usabilibity engineering to the development of a personalised decision support system for Type 1 diabetes self-management. In: The Official Journal of ATTD Advanced Technologies \& Treatments for Diabetes Conference Berlin, Germany-February 20-23, 2019 Diabetes Technology \& Therapeutics. Mary Ann Liebert Inc; 2019. p. ATTD19-0303.

16. Herrero P, López B, Martin C. PEPPER: Patient Empowerment Through Predictive Personalised Decision Support. In: Artificial Intelligence for Diabetes 1st ECAI Workshop on Artificial intelligence for Diabetes at the 22nd European Conference on Artificial Intelligence (ECAI 2016). 2016. p. 8-10.

17. Arduser L. Impatient patients: a DIY usability approach in diabetes wearable technologies. Commun Des Q Rev. 2018;5(4):31-9.

18. Omer T. Empowered citizen "health hackers" who are not waiting [Internet]. Vol. 14, BMC Medicine. BioMed Central Ltd.; 2016. p. 118.

19. Moscardó V, Giménez M, Oliver N, Hill NR. Updated Software for Automated Assessment of 
Glucose Variability and Quality of Glycemic Control in Diabetes. Diabetes Technol Ther. 2020; dia.2019.0416.

20. Torrent-Fontbona F. Adaptive basal insulin recommender system based on Kalman filter for type 1 diabetes. Expert Syst Appl. 2018;101:1-7.

21. Reddy M, Pesl P, Xenou M, Toumazou C, Johnston D, Georgiou P, et al. Clinical safety and feasibility of the advanced bolus calculator for type 1 diabetes based on case-based reasoning: A 6-week nonrandomized single-arm pilot study. Diabetes Technol Ther. 2016;18(8):487-93.

22. Tyler NS, Mosquera-Lopez CM, Wilson LM, Dodier RH, Branigan DL, Gabo VB, et al. An artificial intelligence decision support system for the management of type 1 diabetes. Nat Metab. 2020;2(7):612-9.

23. Tyler NS, Jacobs PG. Artificial intelligence in decision support systems for type 1 diabetes. Sensors (Switzerland). 2020;20(11).

24. Breton MD, Patek SD, Lv D, Schertz E, Robic J, Pinnata J, et al. Continuous Glucose Monitoring and Insulin Informed Advisory System with Automated Titration and Dosing of Insulin Reduces Glucose Variability in Type 1 Diabetes Mellitus. Diabetes Technol Ther. 2018;20(8):531-40.

25. Nimri R, Dassau E, Segall T, Muller I, Bratina N, Kordonouri O, et al. Adjusting insulin doses in patients with type 1 diabetes who use insulin pump and continuous glucose monitoring: Variations among countries and physicians. Diabetes, Obes Metab. 2018;20(10):2458-66. 
Table 1: Baseline demographics for ITT population. PEPPER/Control group started with the PEPPER intervention and Control/PEPPER started with Control.

Results are expressed as median (IQR)/ n (\%). BMI, body mass index; CGM, continuous glucose monitoring; CSII, continuous subcutaneous insulin infusion; DKA, diabetic ketoacidosis; ITT, intention-to-treat; MDI, multiple daily injections; TSH, thyroid stimulating hormone.

\begin{tabular}{|c|c|c|c|}
\hline Demographics & $\begin{array}{l}\text { ITT cohort } \\
(n=54)\end{array}$ & $\begin{array}{l}\text { PEPPER/Control } \\
(\mathrm{n}=24)\end{array}$ & $\begin{array}{c}\text { Control/PEPPER } \\
(\mathrm{n}=\mathbf{2 6})\end{array}$ \\
\hline Gender (female) & $28(51.8 \%)$ & $13(54 \%)$ & $13(50.0 \%)$ \\
\hline Age (years) & $41.5(32.3-49.8)$ & $42.0(37.8-48.0)$ & $41.0(32.8-49.8)$ \\
\hline $\begin{array}{l}\text { BMI }\left(\mathrm{kg} / \mathrm{m}^{2}\right) \\
\text { Height }(\mathrm{cm}) \\
\text { Weight }(\mathrm{kg})\end{array}$ & $\begin{array}{c}26.0(23.8-29.2) \\
169.0(162.4-177.4) \\
72.9(65.1-86.2)\end{array}$ & $\begin{array}{c}26.3(23.9-28.0) \\
169.3(161.5-174.0) \\
71.6(64.9-83.0)\end{array}$ & $\begin{array}{c}25.8(23.2-29.9) \\
170.0(163.1-178.3) \\
74.2(66.0-86.2)\end{array}$ \\
\hline Duration of diabetes (years) & $21.0(11.5-26.0)$ & $22.0(13.0-27.5)$ & $17.5(11.0-24.0)$ \\
\hline $\begin{array}{l}\text { Hypoglycaemia awareness } \\
\text { Gold/Clarke score }<4 \text { ) }\end{array}$ & $54(100 \%)$ & $24(100 \%)$ & $26(100 \%)$ \\
\hline CSII: MDI users & $28: 26$ & $11: 13$ & $13: 13$ \\
\hline $\begin{array}{ll}\text { Baseline use of CGM } & \\
& \text { CSII user } \\
\text { MDI user }\end{array}$ & $\begin{array}{l}3(5.6 \%) \\
1(1.9 \%)\end{array}$ & $\begin{array}{l}1(4.2 \%) \\
0(0.0 \%)\end{array}$ & $\begin{array}{l}2(7.7 \%) \\
1(3.8 \%)\end{array}$ \\
\hline Baseline use of Freestyle Libre & $4(7.4 \%)$ & $2(8.3 \%)$ & $2(7.7 \%)$ \\
\hline $\begin{array}{l}\text { HbAlc (mmol/mol) } \\
\text { HbA1c (\%) }\end{array}$ & $\begin{array}{l}61.0(58.0-66.1) \\
7.7(7.5-8.2)\end{array}$ & $\begin{array}{l}61.0(58.8-67.1) \\
7.7(7.5-8.3)\end{array}$ & $\begin{array}{l}59.3(56.0-66.0) \\
7.6(7.3-8.2)\end{array}$ \\
\hline $\begin{array}{l}\% \text { of participants with previous } \\
\text { DKA }\end{array}$ & $10(18.5 \%)$ & $6(25.0 \%)$ & $4(15.4 \%)$ \\
\hline $\begin{array}{l}\text { \% of participants with previous } \\
\text { severe hypoglycaemia episode }\end{array}$ & $11(18.5 \%)$ & $5(20.8 \%)$ & $4(15.4 \%)$ \\
\hline $\mathrm{TSH}(\mathrm{mU} / \mathrm{L})$ & $1.7(1.1-2.3)$ & $1.8(1.2-2.3)$ & $1.7(1.2-2.3)$ \\
\hline $\begin{array}{l}\text { Total cholesterol }(\mathrm{mg} / \mathrm{dl}) \\
\text { Total cholesterol }(\mathrm{mmol} / \mathrm{l})\end{array}$ & $\begin{array}{c}172(158-194) \\
4.4(4.1-5.0)\end{array}$ & $\begin{array}{c}174(159-200) \\
4.7(4.4-5.4)\end{array}$ & $\begin{array}{c}171(151-190) \\
4.5(4.2-5.0)\end{array}$ \\
\hline
\end{tabular}

Table 2: Intention-to-treat pooled analysis of glycaemic outcomes. AUC, area under the curve 


\begin{tabular}{|c|c|c|c|c|}
\hline & $\begin{array}{c}\text { RUN-IN } \\
(n=54)\end{array}$ & $\begin{array}{l}\text { CONTROL } \\
(n=40)\end{array}$ & $\begin{array}{c}\text { PEPPER } \\
(n=39)\end{array}$ & $\begin{array}{c}\text { P-value } \\
\text { (PEPPER vs } \\
\text { CONTROL) } \\
\end{array}$ \\
\hline \multicolumn{5}{|c|}{ PRIMARY ENDPOINT } \\
\hline $\begin{array}{l}\text { \% time in range } \\
\quad 3.9-10 \mathrm{mmol} / 1(70-180 \mathrm{mg} / \mathrm{dL})\end{array}$ & $55.0(46.4-65.6)$ & $58.4(49.6-64.3)$ & $62.5(52.1-67.8)$ & 0.27 \\
\hline \multicolumn{5}{|c|}{ SECONDARY ENDPOINTS } \\
\hline $\begin{array}{l}\text { \% time in euglycaemia } \\
\quad 3.9-7.8 \mathrm{mmol} / 1(70-140 \mathrm{mg} / \mathrm{dL})\end{array}$ & $30.5(23.9-39.9)$ & $34.0(24.0-40.1)$ & $34.7(28.7-38.9)$ & 0.70 \\
\hline $\begin{aligned} & \% \text { time in hypoglycaemia } \\
&<3.9 \mathrm{mmol} / 1(<70 \mathrm{mg} / \mathrm{dL}) \\
&<3.3 \mathrm{mmol} / 1(<60 \mathrm{mg} / \mathrm{dL}) \\
&<3.0 \mathrm{mmol} / 1(<54 \mathrm{mg} / \mathrm{dL}) \\
&<2.8 \mathrm{mmol} / 1(<50 \mathrm{mg} / \mathrm{dL}) \\
&<2.5 \mathrm{mmol} / 1(<45 \mathrm{mg} / \mathrm{dL})\end{aligned}$ & $\begin{array}{l}2.7(1.6-5.5) \\
1.4(0.7-3.0) \\
1.0(0.4-1.9) \\
0.8(0.2-1.4) \\
0.5(0.1-1.0)\end{array}$ & $\begin{array}{l}2.3(1.1-6.4) \\
0.8(0.4-3.3) \\
0.4(0.1-2.0) \\
0.3(0.1-1.2) \\
0.2(0.0-0.9)\end{array}$ & $\begin{array}{l}2.2(1.5-3.3) \\
0.8(0.5-1.8) \\
0.4(0.2-1.1) \\
0.2(0.1-0.7) \\
0.2(0.0-0.4)\end{array}$ & $\begin{array}{l}0.64 \\
0.69 \\
0.84 \\
0.78 \\
0.78\end{array}$ \\
\hline $\begin{array}{r}\text { \% time in hyperglycaemia } \\
\quad>10 \mathrm{mmol} / 1(>180 \mathrm{mg} / \mathrm{dL}) \\
>15 \mathrm{mmol} / 1(>270 \mathrm{mg} / \mathrm{dL})\end{array}$ & $\begin{array}{c}42.8(30.1-49.3) \\
6.8(3.0-12.6)\end{array}$ & $\begin{array}{l}38.6(30.6-48.0) \\
\quad 4.9(2.7-9.4)\end{array}$ & $\begin{array}{l}35.2(29.8-43.9) \\
4.5(2.2-7.5)\end{array}$ & $\begin{array}{l}0.40 \\
0.50\end{array}$ \\
\hline $\begin{array}{r}\text { Glycaemic variability measures } \\
\text { Mean } \\
\text { Standard deviation } \\
\text { CV (\%) } \\
\text { CONGA } \\
\text { LI } \\
\text { JINDEX } \\
\text { LBGI } \\
\text { HBGI } \\
\text { GRADE } \\
\text { GRADE - \% Hypo } \\
\text { GRADE - \% Eugly } \\
\text { GRADE - \% Hyper } \\
\text { MODD } \\
\text { MAGE } \\
\text { ADDR } \\
\text { M-VALUE } \\
\text { MAG } \\
\text { PGS } \\
\text { IGC } \\
\text { GVP }\end{array}$ & $\begin{array}{c}9.6(8.6-10.3) \\
3.4(3.0-3.9) \\
35.9(33.9-39.6) \\
3.8(3.2-4.3) \\
9.2(6.7-12.0) \\
55.1(44.3-65.0) \\
0.7(0.5-1.4) \\
9.0(6.4-11.6) \\
9.9(8.1-11.7) \\
3.8(1.5-8.2) \\
5.7(3.9-7.9) \\
89.9(85.2-93.6) \\
3.9(3.4-4.5) \\
7.7(7.0-9.0) \\
49.3(43.3-57.3) \\
13.3(10.7-18.5) \\
3.5(3.1-4.5) \\
19.7(16.7-23.7) \\
3.1(2.5-4.0) \\
61.1(48.5-86.5)\end{array}$ & $\begin{array}{c}9.2(8.8-10.1) \\
3.3(2.9-3.9) \\
35.6(31.8-40.7) \\
3.4(2.9-4.0) \\
7.9(5.7-10.8) \\
50.9(43.9-60.0) \\
0.6(0.4-1.5) \\
8.0(6.3-10.5) \\
9.3(7.9-11.0) \\
2.1(1.1-8.5) \\
6.3(4.0-8.6) \\
88.5(84.0-93.5) \\
3.7(3.2-4.2) \\
7.3(6.5-9.1) \\
44.3(41.0-53.9) \\
11.8(9.1-17.0) \\
3.5(2.8-3.8) \\
18.6(15.3-21.7) \\
2.8(2.2-3.8) \\
59.9(43.1-70.1)\end{array}$ & $\begin{array}{c}9.0(8.7-9.7) \\
3.1(2.8-3.6) \\
34.6(31.6-38.0) \\
3.5(3.0-4.0) \\
8.1(5.8-10.3) \\
48.6(43.7-56.5) \\
0.6(0.4-1.0) \\
7.6(6.2-9.8) \\
8.7(7.7-10.4) \\
2.3(1.4-4.8) \\
7.5(5.0-9.3) \\
89.8(85.2-92.1) \\
3.5(3.3-4.0) \\
7.3(6.5-8.3) \\
42.0(38.6-51.6) \\
10.0(8.1-13.6) \\
3.3(2.8-4.0) \\
16.2(14.3-21.1) \\
2.4(1.9-3.0) \\
55.4(44.4-74.2)\end{array}$ & $\begin{array}{l}0.56 \\
0.40 \\
0.43 \\
0.91 \\
0.91 \\
0.42 \\
0.71 \\
0.40 \\
0.32 \\
1.00 \\
0.25 \\
0.98 \\
0.58 \\
0.56 \\
0.38 \\
0.22 \\
0.87 \\
0.18 \\
0.21 \\
0.95\end{array}$ \\
\hline $\begin{array}{r}\text { Secondary Glycaemic Outcomes } \\
\text { Mean Glucose } 1 \mathrm{hr}(\mathrm{mmol} / \mathrm{L}) \\
\text { Mean Glucose } 2 \mathrm{hr}(\mathrm{mmol} / \mathrm{L}) \\
\text { Mean AUC }(\min \times \mathrm{mmol} / \mathrm{L})\end{array}$ & $\begin{array}{c}10.3(9.4-11.3) \\
10.4(9.2-11.3) \\
2215.9(2024.8- \\
2395.9)\end{array}$ & $\begin{array}{c}9.9(9.2-11.6) \\
9.8(9.1-11.4) \\
2081.1(2035.5- \\
2413.5)\end{array}$ & $\begin{array}{c}10.0(9.0-10.6) \\
9.9(9.9-10.7) \\
2160.8(1970.2- \\
2243.5)\end{array}$ & $\begin{array}{l}0.79 \\
0.84 \\
0.92\end{array}$ \\
\hline Mean minimum glucose $(\mathrm{mmol} / \mathrm{L})$ & $6.8(6.1-7.5)$ & $6.8(6.1-7.5)$ & $6.7(6.0-7.3)$ & 0.75 \\
\hline $\begin{array}{l}\text { Number of hypo episodes } \\
\text { Postprandial hypo episodes }(5 \mathrm{hr})\end{array}$ & $\begin{array}{l}4.5(2.0-7.0) \\
1.0(0.0-2.0)\end{array}$ & $\begin{array}{l}2.0(1.0-7.0) \\
1.0(0.0-1.0)\end{array}$ & $\begin{array}{l}2.0(1.0-4.0) \\
0.0(0.0-1.0)\end{array}$ & $\begin{array}{l}0.62 \\
0.75\end{array}$ \\
\hline $\begin{array}{r}\text { HbAlc (mmol/mol) } \\
\text { HbAlc }(\%) \\
\text { Weight }(\mathrm{kg}) \\
\text { Basal insulin (units) }\end{array}$ & $\begin{array}{c}61.0(57.5-66.1) \\
7.7(7.5-8.2) \\
72.9(65.1-86.2) \\
21.6(18.0-27.7)\end{array}$ & $\begin{array}{c}58.5(53.0-63.4) \\
7.5(7.0-7.9) \\
73.9(64.8-84.9) \\
25.9(18.0-29.2)\end{array}$ & $\begin{array}{c}58.5(54.0-61.9) \\
7.5(7.1-7.7) \\
75.0(66.7-85.0) \\
25.0(18.5-29.2)\end{array}$ & $\begin{array}{l}0.82 \\
- \\
0.81 \\
0.76\end{array}$ \\
\hline
\end{tabular}


Table 3: Quality of life questionnaire scores in the intention-to-treat population. PAID, problem areas in diabetes (scored out of 100 with higher scores indicating distress); DTSQs, diabetes treatment satisfaction questionnaire (scored out of 36 indicating very satisfied); DTSQs perceived frequency of hypo- and hyperglycemia are scored from 0 (none of the time) to 6 (most of the time); DQOL, diabetes quality of life (based on Likert 5-point scale from 1 to 5 with high scores indicate dissatisfaction, frequent impact, or frequent worry). ${ }^{+}$Run-in $n=53$; ${ }^{++}$Run-in $\mathrm{n}=53$, control $\mathrm{n}=39 .{ }^{*} \mathrm{p}<0.05$ indicates significance.

\begin{tabular}{|c|c|c|c|c|}
\hline & $\begin{array}{c}\text { RUN-IN } \\
(n=54)\end{array}$ & $\begin{array}{c}\text { CONTROL } \\
(n=40)\end{array}$ & $\begin{array}{c}\text { PEPPER } \\
(n=39)\end{array}$ & $\begin{array}{c}\text { P-value } \\
\text { (PEPPER vs } \\
\text { CONTROL) }\end{array}$ \\
\hline PAID Questionnaire ${ }^{+}$ & $18.8(11.3-31.3)$ & $15.6(9.7-24.4)$ & $17.5(10.0-28.8)$ & 0.44 \\
\hline $\begin{array}{l}\text { DTSQs } \\
\text { Global Score } \\
\text { Perceived hypoglycaemia } \\
\text { Perceived hyperglycaemia }\end{array}$ & $\begin{array}{c}26(24-30) \\
2(1-3) \\
2(1-3)\end{array}$ & $\begin{array}{c}32(28-33) \\
2(1-2) \\
2(1-2)\end{array}$ & $\begin{array}{c}31(28-34) \\
2(2-4) \\
2(2-4)\end{array}$ & $\begin{array}{c}0.83 \\
0.03^{*} \\
0.32\end{array}$ \\
\hline $\begin{array}{r}\text { DQOL }^{++} \\
\text {Global Score } \\
\text { Satisfaction } \\
\text { Impact } \\
\text { Worry: Social/Vocational } \\
\text { Worry: Diabetes Related }\end{array}$ & $\begin{array}{l}1.9(1.5-2.4) \\
2.1(1.9-2.5) \\
2.5(1.6-3.3) \\
1.3(1.1-1.9) \\
2.0(1.5-2.5)\end{array}$ & $\begin{array}{l}1.7(1.3-2.1) \\
1.9(1.6-2.3) \\
2.0(1.5-2.7) \\
1.4(1.1-1.9) \\
1.8(1.5-2.3)\end{array}$ & $\begin{array}{l}1.7(1.4-2.0) \\
1.8(1.4-2.2) \\
2.1(1.6-2.9) \\
1.4(1.2-1.6) \\
2.0(1.5-2.4)\end{array}$ & $\begin{array}{l}0.80 \\
0.29 \\
0.49 \\
0.84 \\
0.29\end{array}$ \\
\hline
\end{tabular}


Table 4: Intention-to-treat pooled analysis of safety system outcomes for last 28 days of each intervention phase. Carbohydrate $(\mathrm{CHO})$ recommendations refer to a personalized oral dose of carbohydrates (based on weight) to revert hypoglycaemia and minimize rebound hyperglycaemia. \% partial and \% total suspension reported as proportions of the total time frame (the remaining time was not in suspension). No safety system data was available for 1 participant from the ITT analysis. Data presented as median (IQR) per participant.

\begin{tabular}{|c|c|c|c|}
\hline & $\begin{array}{c}\text { CONTROL } \\
(\mathbf{n = 3 9 )} *\end{array}$ & $\begin{array}{c}\text { PEPPER } \\
\mathbf{( n = 3 8 )}\end{array}$ & $\begin{array}{c}\text { P-value } \\
\text { (PEPPER vs } \\
\text { Control) }\end{array}$ \\
\hline $\begin{array}{r}\text { Safety System } \\
\text { Total bolus insulin (units/day) }\end{array}$ & $32.8(23.9-53.5)$ & $32.6(22.1-51.0)$ & 0.96 \\
No. of hypo alarms/day & $1.5(0.9-2.4)$ & $1.3(1.0-2.0)$ & 0.44 \\
No. of hypo alerts/day & $0.9(0.5-1.3)$ & $0.9(0.6-1.1)$ & 0.53 \\
$1.5(1.0-1.9)$ & $1.5(1.1-2.0)$ & 0.78 \\
No. of CHO recommendations/day & $3.2(0.8-6.8)$ & $2.6(1.1-4.6)$ & 0.74 \\
Mean CHO recommendation (g) & $13.8(10.4-17.0)$ & $12.1(9.9-14.5)$ & 0.16 \\
For PUMP users & & & 0.54 \\
Number of suspensions/day & $1.1(0.9-1.4)$ & $1.2(1.0-1.5)$ & 0.41 \\
\% of total time in partial suspension & $2.7(2.3-3.4)$ & $2.5(2.0-3.4)$ & 0.63 \\
\% of total time in total suspension & $2.3(1.9-3.7)$ & $2.9(2.2-3.5)$ & \\
\hline
\end{tabular}


Figure 1: Overview of the components of the PEPPER system architecture. (A) PEPPER user platform, which displays glucose trends, activity data and quick access to the insulin recommender. (B) Bolus advisor which requests additional lifestyle information from the user in addition to the carbohydrate content of the meal to provide adaptive insulin dose recommendations. (C) Revisions of case outcomes and case adaptations are performed with the PEPPER clinical platform.

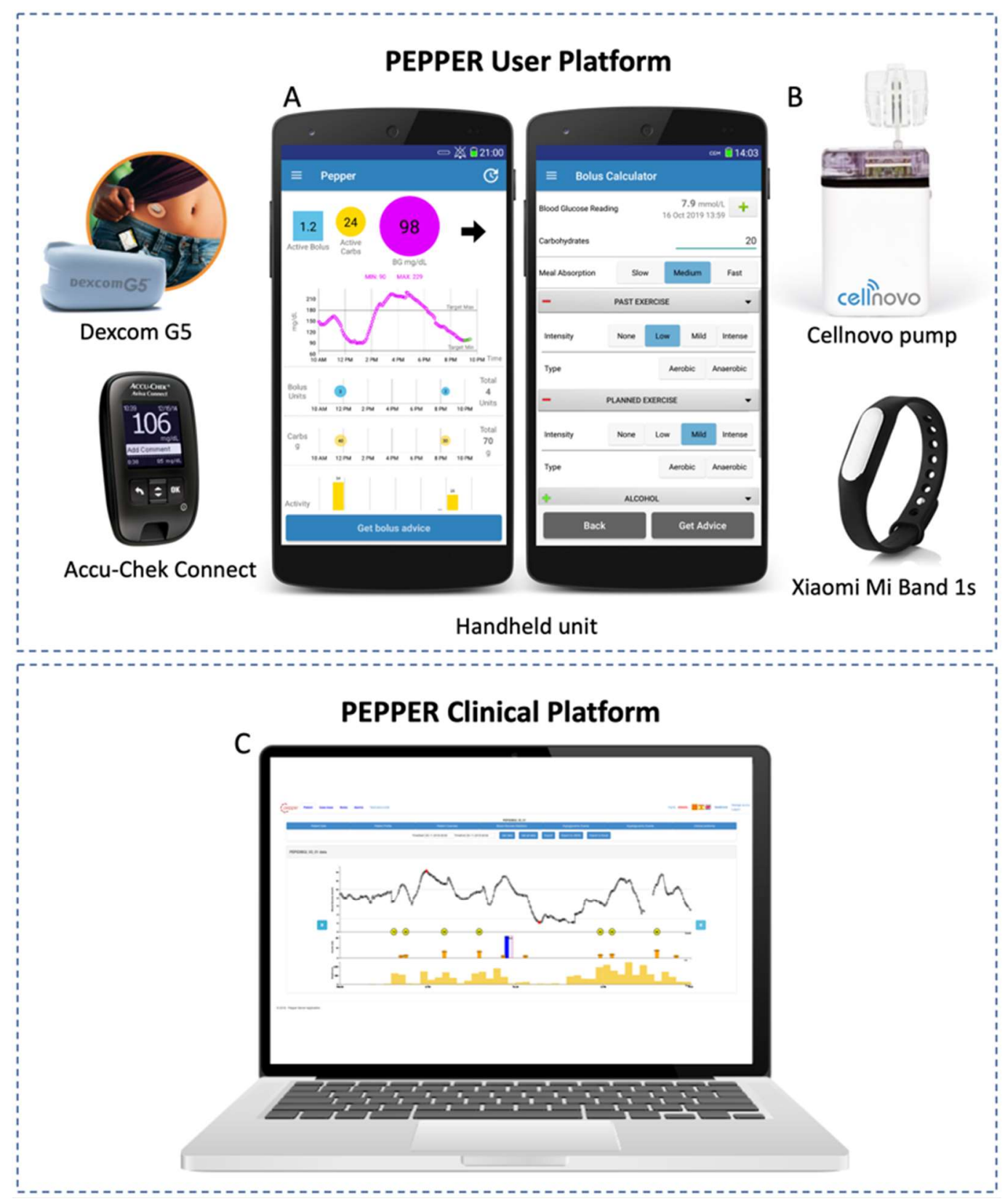


Figure 2: Consort Flow Diagram. The ITT study population included 54 participants completing the run-in period. For the PPP analysis, 33 participants were included. Reasons for exclusion were as follows: early study termination due to Cellnovo withdrawal $(n=10)$; participants declining to participate $(n=8)$; $\operatorname{SAE}(n=2)$; completion of study but non-use of PEPPER adaptive bolus advisor $(n=1)$.

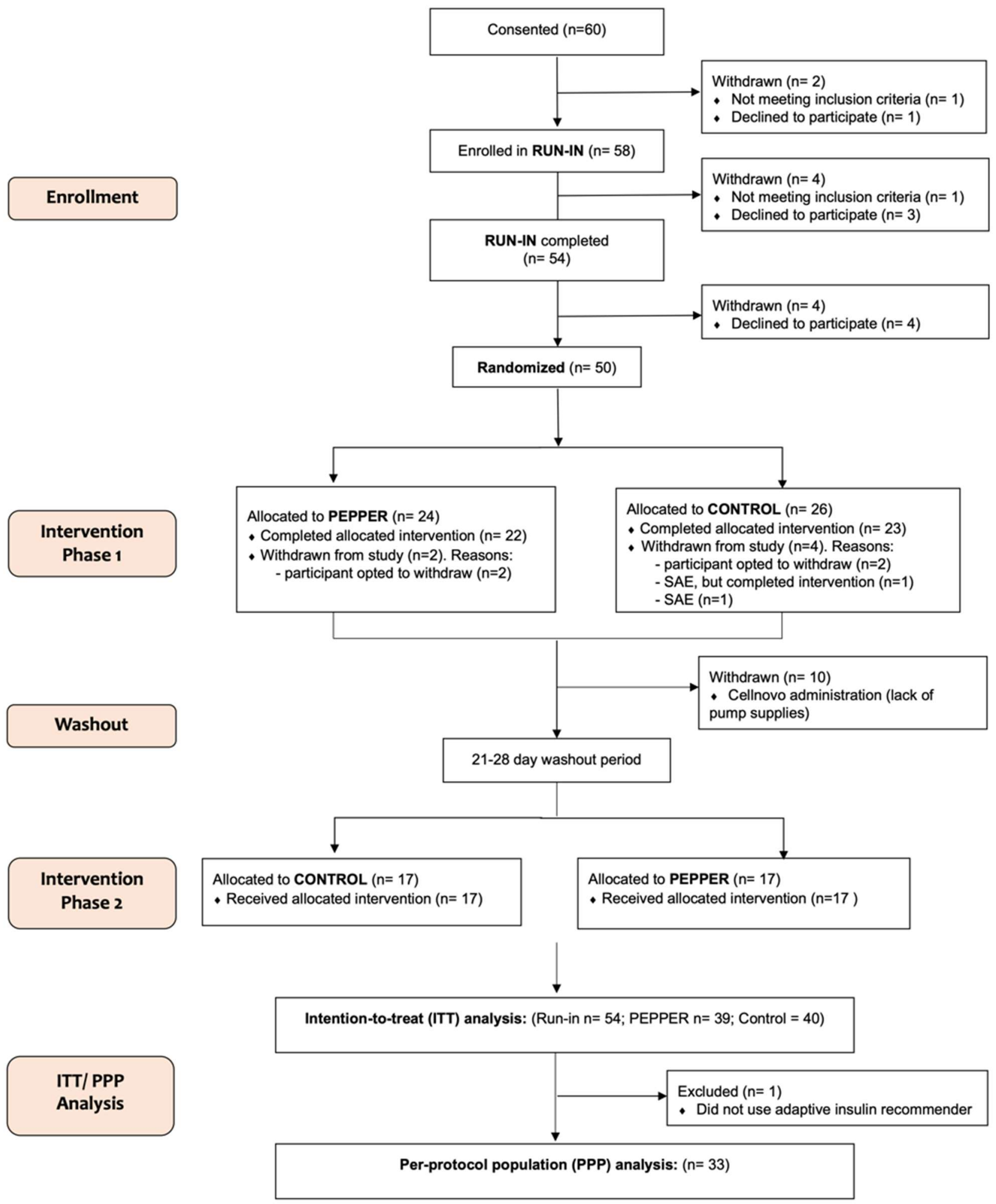

